\title{
"Founding a League of United Latin American Citizens (LULAC) council in a U.S. medical school: A new blueprint for Latino medical student organizations to enhance their public health outreach efforts."
}

Santiago R Gonzalez, ${ }^{1}$ Basilia Gonzalez, ${ }^{2}$ Rebecca Moreira, ${ }^{3}$ Raj Singh, ${ }^{4}$ Billy Thomas, MD, MPH,,${ }^{5}$ Sara Tariq, MD, ${ }^{6}$ Jasna Vuk, MD, PhD $^{7}$

\begin{abstract}
Background: In the next 40 years, the Hispanic population will represent $29 \%$ of the U.S. population. Hispanics living in the U.S. face cultural and economic barriers to receiving healthcare. To address the health disparities faced by Hispanic populations at the local level, an inter-professional group of Hispanic students from the University of Arkansas for Medical Sciences (UAMS) founded the first League of United Latin American Citizens (LULAC) at an academic medical center.
\end{abstract}

Methods: This article represents a descriptive guide to the methods we utilized to establish the organization, the health fairs, a mentoring program, and a medical Spanish curriculum.

Additionally, this article demonstrates how our partnership with LULAC facilitated each of the activities we organized by enhancing public awareness, increasing available resources, and enabling community partnerships with local businesses and media outlets.

Results: Since the organization was established in 2017, 87 students from the Colleges of Medicine, Pharmacy, Nursing, and Health Professions have become UAMS LULAC members. Together they screened 194 uninsured Hispanic patients at health fairs, mentored pre-medical students at local colleges, and designed and implemented the curriculum for a medical Spanish course in which 64 students participated.

Conclusions: Becoming a LULAC council is an innovative and highly beneficial avenue for both new and existing Hispanic medical student organizations. By following a similar methodology, and by partnering with local LULAC councils and their pre-existing networks, Hispanic student organizations may also increase their access to support, resources, and volunteers to ultimately enhance their educational experience and public health outreach efforts.

\footnotetext{
* Correspondence: sgonzalez@uams.edu

${ }^{\mathbf{1}}$ Founding President of UAMS LULAC, MD, MPH Candidate, Medical Student, University of Arkansas for Medical Sciences, College of Medicine, Little Rock, AR, USA.

Full list of author information is available at the end of the article
} 


\section{Introduction}

In the next 50 years, the Hispanic population will represent $29 \%$ of the United States (U.S.) population (1). There are 54 million Hispanics living in the US, which makes them the largest minority group in the country (2). Hispanics living in the U.S. are disproportionally affected by health conditions such as obesity, diabetes, nonalcoholic fatty liver disease, and unintentional injuries (2-5). Lifestyle decisions and cultural differences may not be sufficient to explain the source of health disparities experienced by these populations. Access to medical screenings, follow-up medical care, culture, and socioeconomic status have all been shown to play a significant role in individual and population health (6).

Recognizing the hardships that many Hispanics face, students at the University of Arkansas for Medical Sciences (UAMS) in Little Rock, Arkansas, sought to make a change in the local community. In January 2017, an inter-professional group of students from the colleges of Medicine, Nursing, Pharmacy, and Health Professions at UAMS founded the first-ever League of United Latin American Citizens (LULAC) council centered in a U.S. medical school.

\section{History of Hispanic student organizations}

Hispanic medical student organizations have been around since the 1970s (7-10). The National Boricua Latino Health Organization (NBLHO) was founded in 1972 at Harvard Medical School (7), The National Network of Latin American Medical Students (NNLAMS) was founded in 1987 (8), and the National Hispanic Medical Association (NHMA) in 1994 (9). All three of these organizations were later unified under one name, the Latino Medical Student Organization (LMSA) in 2010 (10).

Although Hispanic medical student organizations have been active for decades, there are no reports in the literature about the methods they have used to found their organizations, and to organize their activities. Through this article, we do not seek to create a separate entity from the previously-founded organizations, but rather, we would like to demonstrate the benefits of partnering with LULAC and its ever-growing network of members.

\section{Why LULAC?}

LULAC was founded in Harlingen, Texas, in 1929, and with 132,000 members and over 1100 councils throughout the United States and Puerto Rico, it is the largest and oldest Hispanic organization in the United States (11). The UAMS LULAC members collaborated with three previously established LULAC councils in central Arkansas, including the University of Arkansas of Little Rock LULAC, the Little Rock LULAC Council 750, and the LULAC Council of Faulkner County, in order to reach a more significant number of Hispanics in the area.

This unique partnering with a national Hispanic organization such as LULAC is beneficial for many reasons. For example, the Hispanic community already knows and trusts LULAC which immediately gives the student organization a level of credibility that would otherwise take a long time to develop. Also by joining with LULAC, we had access to networking groups who have been in the central Arkansas area for more than 35 years. These groups have been able to connect with local government, including the Little Rock mayor and superintendents. They have scholarship and financial aid programs, and most importantly, they have a 
large number of volunteers who are passionate about serving the local Latino communities. Healthcare of Latinos is strongly related to their social determinants of health, such as socioeconomic status, educational opportunities, personal growth, immigration status, access to insurance, etc. (2-6). Taking this into account, we decided to join an organization such as LULAC that focuses on all these aspects of Hispanic advancement in addition to the delivery of healthcare.

\section{Methods}

\section{Founding the organization}

Our student organization was sponsored by the UAMS Center for Diversity Affairs (CDA) and by four faculty members who are firmly committed to diversity and inclusion with the goal of establishing the long-term stability of the organization. The CDA goals of increasing diversity at our institution are closely aligned with the goals of LULAC councils in central Arkansas in terms of promoting educational opportunities for high school and college level students. In fact, for the past 10 years, the CDA has donated money toward LULAC scholarships and has participated in their scholarship fundraising events to attract Latino students to UAMS. We registered the organization with both the national LULAC headquarters and the UAMS Office of Student Life. To obtain non-profit status, we registered our organization as a 501(c)(4) organization. To address the health care needs of the local Hispanic population, UAMS LULAC organized free community health fairs, created mentoring programs, and designed and implemented a structured medical Spanish course.

\section{Health fairs}

By becoming a LULAC council, the logistics associated with organizing community health fairs were facilitated immensely. To make sure that we tailored our health fair to the needs of our local Hispanic population, we performed a needs assessment at a large Catholic church in Central Arkansas where approximately 1400 Hispanic people attend to on a weekly basis. The majority of participants indicated a lack of insurance, affordability of doctor's visits, language barriers, lack of knowledge on how to make an appointment, and other factors as barriers to receiving health care (12). These individuals indicated that the only way they can be seen by a healthcare provider is through health fairs or other community events; this response informed our Health Fair structure (12). Studies have shown increased participation by holding health fairs after church services, so we organized both health fairs at the same church in which we held the needs assessment (13).

Our connections with LULAC aided us in the process of securing a grant for health screening supplies from the Arkansas Minority Health Commission (14). Our local LULAC network also helped us connect with El Latino newspaper, a local publication widely read by the Latino community in central Arkansas. El Latino included announcements and did a report about our health fair to raise awareness and increase participation within the local Latino community (15). Our affiliation with LULAC also helped us get in touch us with a local bank who provided free snacks for health fair participants.

We recruited 54 LULAC members and additional students from all colleges at UAMS to participate as volunteers in the two health fairs. Although student providers 
have the tendency to self-select, we chose volunteers based on interest, past volunteer efforts with LULAC, time and availability. Prior to participating, we oriented students with an in-services outlining of the patient population (racial and ethnic groups and socioeconomic status), individual and population health status, common comorbidities, and the design of patient intake (triage, vital signs, laboratory draws). We also taught them the importance of both counseling patients and scheduling follow up at the local free clinics. Through this experience, we aimed to increase student awareness and to mitigate some of the effects of implicit bias on the basis of race, ethnicity, and English as a second language on patient care and outcomes (16).

We set up stations to screen blood pressure, basal metabolic index (BMI), blood glucose, and cholesterol. Three licensed physicians counseled participants on the results of their screenings and provided a list of local free health clinics for follow-up appointments.

\section{Mentoring program}

Due to the large network of local LULAC councils, which are largely centered at local universities, we were able to reach a larger number of potential mentees interested in pursuing a career in healthcare. Academic and career mentoring for underrepresented minorities have been shown to have a positive impact on the inclusion, productivity, and career success of mentees (17). We developed a mentoring program to provide role models and guidance for Hispanic students in the local elementary, middle, and high schools. We also designed the mentoring program to increase diversity in the matriculating classes of students at UAMS, which we aimed to achieve by providing support to ethnically diverse college students interested in pursuing a career in the health care field. We recruited students from all colleges to work as mentors. We instructed teachers, parents, or students who were interested in pairing a student with a mentor to contact the LULAC secretary. After obtaining the mentee's information, we assigned a mentor who would best fit the needs and expectations of the student mentee. Once the pairing was established, the specific needs and goals of the mentee determined the extent of the mentoring experience.

Being part of the LULAC network helped us connect with students interested in pursuing a career in health care. Currently, there are three undergraduate universities in central Arkansas with an active LULAC council. Students at these undergraduate institutions have an opportunity to interact with faculty and student members from our medical center while working at the various community events we host. Through the LULAC network, students interested in pursuing a career in the medical field are able to easily reach out to our Latino members, who may share similar experiences and who may have faced the same struggles the undergraduate students are currently going through.

\section{Medical Spanish course}

Literature has demonstrated a significant connection between limited English proficiency and poorer health outcomes $(18,19)$. In addition, patients who experience language barriers often report worse medication adherence and lower satisfaction with their medical care $(20,21)$. To ensure that our course met student needs and expectations, we designed a formal needs assessment survey of students at our institution. A total of 698 responses were obtained from students across all colleges (22). The survey results strongly indicated that UAMS students wanted opportunities to learn medical Spanish. However, at the time no structured, longitudinal medical Spanish 
course was offered. To address these issues, we designed a course to promote a basic understanding of medical Spanish and cultural competency. We launched the course in September 2018, and UAMS LULAC membership was a requirement to participate. Our medical Spanish program is unique in that the curriculum is designed through a case-based approach and each lesson centers on a common chief complaint. Partnership with the national LULAC organization increased our course's credibility with students, who were eager to sign up for LULAC membership to participate in the course.

Through our LULAC network, we were able to recruit two certified medical Spanish instructors with previous experience teaching medical Spanish courses. We supplemented our curriculum with a textbook titled: Complete Medical Spanish: Practical Medical Spanish for Quick and Confident Communication, which we selected for its inclusion of Spanish grammar, targeted clinical relevance, and cultural correlations (23). In the first few months, students were familiarized with basic vocabulary that would allow them to introduce themselves to Spanish-speaking patients. We also taught them the proper way to work with interpreters to ensure that their messages were accurately transferred to the patient. We designed the remaining lessons to help students learn how to ask about the chief complaint, take a full history, and conduct a physical exam. We incorporated interactive features in which participants were asked to pronounce words out loud and converse with one another through various exercises. In each class, we also included relevant cultural competency training to help students understand how culture affects patients' expectations about healthcare. For example, we made students aware of the differences in the way that Hispanic patients tend to portray pain.

Once students mastered the Spanish necessary for a general medical encounter, we transitioned the lessons to a case-based curriculum with clinical vignettes focused around common chief complaints like diabetes, hypertension, and pregnancy. In addition to formal bimonthly class meetings, we held student-led conversation hours on alternating weeks to give students an opportunity to practice previous material. Students who self-identified as having an advanced level of Spanish fluency facilitated these meetings. This way, the facilitators could answer questions, correct errors, and engage in conversational Spanish with their peers.

\section{Results}

Since its origin, a total of 87 students from the Colleges of Medicine, Pharmacy, Nursing, and Health Professions signed up for membership in UAMS LULAC. By creating an inter-professional organization, we were able to cater to a wider variety of needs in the Hispanic community. For example, by collaborating with the Dental Hygiene program, we were able to provide dental services to patients who attended the health fairs.

In the two health fairs combined, we screened 194 uninsured Hispanic patients. The patients were very receptive to our medical outreach events and were grateful to have medical professionals bring healthcare services to their community. Multiple patients inquired about the next time a health fair would be held with the intent of bringing family and friends. 
Through the mentoring program, we were able to present multiple career options and guidance to a large number of Hispanic students and their parents. As the mentoring program progressed, the mentors were invited to speak to two classes of elementary students, and pre-medical students from local colleges were paired with LULAC mentors in the College of Medicine.

In the medical Spanish class, 64 students signed up for UAMS LULAC membership to enroll in the class, and 53 purchased a textbook as a course companion. Of these students, a total of $53(83 \%)$ were from the College of Medicine, 7 (11\%) from the College of Pharmacy, 3 (5\%) from the College of Health Professions, and $1(2 \%)$ from the College of Nursing. The number of students participating from different colleges reflects the class size of the respective programs, with the College of Medicine being the largest program at UAMS. For example, the Physicians Assistant program in the College of Health Professions has a class size of 39 per year for two years, whereas the College of Medicine class size is 174 per year for four years. The robust participation of students from various UAMS programs illustrates their awareness of the importance of communication in the access and delivery of quality health care.

\section{Discussion}

Over half of UAMS LULAC student members are not of Hispanic descent. Nonetheless, they are able to relate to Hispanic patients regardless of culture and language differences, evidenced by their volunteer hours at Health Fairs and mentoring programs and their participation in the medical Spanish course. These students prove that individuals of different ethnicities can come together to help patients regardless of the background of the patient or the healthcare provider.
Ultimately, the desire to provide answers and solutions for patients overcame any ethnic difference.

Through our experience, we learned that organizations in our community are willing to contribute, provide services, and host community events. In many cases, community members were not aware of the many free resources and healthcare opportunities available in the area. In the future, a large part of our work will focus on outreach and informing patients of local resources and support services. By creating a blueprint for the organization that clearly outlines the roles and responsibilities of officers and volunteers, the organization will be sustainable. This, along with the continued support of the Center for Diversity Affairs, will allow ease of transition as leadership changes. We plan to expand our mentoring program not only to those who reside in nearby communities but also to any Hispanic student interested in applying to our institution. It is our goal that these efforts lead to an increase in diversity of the student body at our institution.

The UAMS LULAC council is a student-led organization which was founded with the aim of promoting the health care advancement of underserved Hispanic communities in central Arkansas. The organization has successfully recruited members, advisors, volunteers, and community supporters merely by taking the time to speak about health disparities faced by Hispanics in the U.S. and to create an avenue for people to become a part of the solution.

Our organization highlights the importance of associating with other community organizations that promote Latino healthcare and overall personal advancement, such as the national LULAC organization. By taking 
a similar approach, other Latino student organizations at U.S. institutions also can take advantage of the large LULAC network. Although LULAC councils may not be present everywhere, the benefit of association with LULAC network and resources can still be advantageous. The founding members and advisors of UAMS LULAC hope the work done thus far influences other U.S. medical schools to partner with LULAC, so they, too, can leverage the many resources and networking opportunities that this national organization can provide for their institutions.

\begin{abstract}
Author Contributions Statement
SG is the Founding President of UAMS LULAC and he contributed by establishing the organization, planning events, writing the first draft of the manuscript including the abstract, methods, results, and discussion sections of the paper, data gathering, data analysis, editing, formatting, provided substantial ideas to develop the manuscript, and revised all sections of the paper. BG is the Founding Vice President of UAMS LULAC and she contributed to the manuscript by establishing the organization, planning events, revising and writing parts of the manuscript including the abstract, methods, results, and discussion sections of the paper, data gathering, data analysis, editing, and formatting. RM is the medical Spanish Coordinator and she contributed to this manuscript by helping to develop the medical Spanish curriculum, writing the medical Spanish section of the manuscript, and helping to revise all sections of the manuscript. RS contributed to the manuscript by helping to revise the manuscript, the literature review, and formatting of the manuscript. BT, ST, and VJ are faculty advisors who provided substantial ideas and guidance to develop the manuscript, supported the development of the organization, and revised all sections of the paper.
\end{abstract}

\section{Conflict of Interest Statement}

The authors declare that the information in this article was presented in the absence of any commercial or financial relationships that could be construed as a potential conflict of interest.

\section{Acknowledgements}

The authors would like to show their appreciation the UAMS CDA staff members Nicholas Pettus, Kimberlyn Blann-Anderson and Odette Woods for their support and guidance throughout the first three years of the organization. The authors are also deeply thankful to the advisors, officers, and members of the surrounding LULAC Councils, the University of Arkansas of Little Rock LULAC, the Little Rock LULAC Council 750, and the LULAC Council of Faulkner County. We would also like to thank the Arkansas Minority Health Commission for their grant that made the health fairs possible. We would also like to thank our advisors Dr. Trevino Richard, Dr. Cesar Compadre, Dr. Erick Messias, and our student officers Kylie Hayes, and Giselle Moran for all their help at the beginning stages of the organization. Lastly, we would like to thank Eduardo Gonzalez, Carolina Gonzalez, Luis Oseguera, and Martha Oseguera for their continuous encouragement, and for teaching us the value of embracing our Hispanic roots and giving back to our communities.

\section{Funding}

No funding was received for the development or publication of this manuscript.

\section{Data Availability Statement}

All datasets generated for this study are included in the manuscript. National reports from the US Census Bureau and the U.S. Department of Health \& Human Services were included in the references section. The files included in this article contained the following copyright information statement: All material appearing in this report is in the public domain and may be reproduced or copied without permission; citation as to source, however, is appreciated.

\section{Ethics statements}

No animal studies are presented in this manuscript. No human studies are presented in this manuscript. No potentially identifiable human images or data is presented in this study.

\section{Contribution to the Field Statement}

In just five decades, the Hispanic population will represent $29 \%$ of the United States population. Health care professionals must be equipped to adequately meet the needs of this rapidly growing Hispanic patient population. Hispanic student organizations can provide future healthcare workers with an opportunity to develop the language skills 
and cultural awareness that they can utilize to meet the needs of the rapidly growing Hispanic population.

Hispanic medical student organizations have been around since 1972, but to the best of our knowledge, there are no reports in the literature describing the methods they used to build successful organizations from the ground up.

This article represents a descriptive guide to the methods and logistics we utilized to establish the first LULAC organization at a U.S. medical school. Additionally, this article demonstrates how our partnership with LULAC facilitated each of the activities we organized by enhancing public awareness, increasing available resources, and enabling community partnerships with local businesses and media outlets.

By partnering with local LULAC councils and their pre-existing networks, other Hispanic medical student organizations can also increase their access to additional support, resources, and volunteers to ultimately enhance their educational experience and public health outreach efforts.

\section{Author Details}

${ }^{1}$ Founding President of UAMS LULAC, MD, MPH Candidate, Medical Student, University of Arkansas for Medical Sciences, College of Medicine, Little Rock, AR, USA.
${ }^{2}$ Founding Vice President of UAMS LULAC, MD Candidate, Medical Student, University of Arkansas for Medical Sciences, College of Medicine, Little Rock, AR, USA.

${ }^{3}$ Medical Spanish Coordinator of UAMS LULAC, MD, MPH Candidate, Medical Student, University of Arkansas for Medical Sciences, College of Medicine, Little Rock, AR, USA.

${ }^{4}$ Certified Research Assistant, Department of Immunology and Microbiology, University of Arkansas for Medical Sciences, Little Rock, AR, USA.

${ }^{5}$ Vice Chancellor for Diversity and Inclusion, and Professor, Department of Neonatology, University of Arkansas for Medical Sciences, College of Medicine in Little Rock, AR, USA

${ }^{6}$ Professor, Department of Internal Medicine, Associate Dean for Undergraduate Education, and the Medical Director of the Center for Clinical Skills Education at the University of Arkansas for Medical Sciences in Little Rock, AR, USA

${ }^{7}$ Associate Professor, Academic Affairs Student Success Center, University of Arkansas for Medical Sciences in Little Rock, AR, USA 


\section{References}

1. Colby SL, Ortman JM. Projections of the size and composition of the U.S. Population: 2014 to 2060. Census Bureau US. Current Population Reports (2015). Available at:

https://www.census.gov/content/dam/Census/library/ publications/2015/demo/p25-1143.pdf

2. Lucas JW, Freeman G, Adams P. Health of Hispanic Adults: United States, 2010-2014. NCHS Data Brief no 251. Hyattsville, MD; National Center for Health Statistics (2016). Available at: https:/www.cdc.gov/nchs/data/databriefs/db251.pdf

3. Hales CM, Carrol MD, Fryar CD, Ogden CL. Prevalence of obesity among adults and youth: United States, 2015-2016. NCHS Data Brief, no 288. Hyattsville, MD: National Center for Health Statistics (2017). Available at:

https://www.cdc.gov/nchs/data/databriefs/db288.pdf

4. Centers-for-Disease-Control-and-Prevention. National diabetes statistics report: estimates of diabetes and its burden in the United States. Atlanta: US Department of Health and Human Services (2017). Available at:

https://www.cdc.gov/diabetes/pdfs/data/statistics/nati onal-diabetes-statistics-report.pdf

5. Smith ML, Bergeron CD, Riggle SD, Meng L, Towne SD, Ahn S Et al. Self-care difficulties and reliance on support among vulnerable middle-aged and older adults with chronic conditions: A crosssectional study. Maturitas (2017) 104:1-10. doi: 10.1016/j.maturitas.2017.06.030

6. Miranda-Diaz C, Betancourt E, Ruiz-Candelaria Y, Hunter-Mellado RF. Barriers for compliance to breast, colorectal, and cervical screening cancer tests among Hispanic patients. Int J Environ Res Public Health (2016) 13(1): 21. doi: 10.3390/ijerph13010021

7. Leonard DJ, Lugo-Lugo CR, eds. Latino History and Culture: An Encyclopedia. Armonk, NY: Sharpe Reference (2010).

8. Rodriguez R. Latino Talent: Effective Strategies to Recruit, Retain, and Develop Hispanic Professionals. Hoboken, N.J: John Wiley \& Sons (2008).

9. National Hispanic Medical Association - NHMA. https://www.nhmamd.org/. Accessed May 12, 2019.
10. LMSA National » About. http://lmsa.net/org. Accessed May 12, 2019.

11. About Us. LULAC. https://lulac.org/about/. Published 2018. Accessed November 29, 2018.

12. Gonzalez SR, Gonzalez B, Hayes K, Davies D, Thomas B. The Founding of an Interprofessional Hispanic Student Organization - Methods and Impact. AAMC Group on Business Affairs, Group on Diversity Affairs, Group on Institutional Planning Joint Meeting. Chicago, Illinois. (2019).

13. Derose, KP; Williams, MV; Branch, CA; Florez, KR; Hawes-Dawson, J; Mata, MA et al. A Community-Partnered Approach to Developing Church-Based Interventions to Reduce Health Disparities Among African-Americans and Latinos. $J$ Racial Ethn Health Disparities (2019) 6(2):254-264. doi: 10.1007/s40615-018-0520-z.

14. Arkansas Minority Health Commission - About Us. http://arminorityhealth.com/about-us/missionvision-goal/. Accessed December 10, 2018.

15. Michael Leidermann. Hundreds of people at the 1st LULAC-UAMS Health

Fair. El Latino Arkansas. http://www.ellatinoarkansas $. \mathrm{com} /$ issueview.cfm?IssueID $=687 \&$ Entry $=$ Archive. $\mathrm{P}$ ublished March 2, 2018. Accessed May 8, 2019.

16. Gonzalez CM, Garba RJ, Liguori A, Marantz PR, McKee MD, Lypson ML. How to Make or Break Implicit Bias Instruction: Implications for Curriculum Development. Acad Med (2018) 93(11S): S74-S81. doi:10.1097/ACM.0000000000002386

17. Hund AK, Churchill AC, Faist AM, Havrilla CA, Stowell S, McCreery HF et al. Transforming Mentorship in STEM by Training Scientists to Be Better Leaders. Can J Chem Eng (2018) 8(20):99629974 doi:10.1002/ece3.4527

18. Vela M, Fritz C, Jacobs EA. Establishing medical students' cultural and linguistic competence for the care of Spanish-speaking limited English Proficient patients. J Racial Ethn Health Disparities (2016) 3(3): 484-88. doi: 10.1007/s40615-015-0165-0.

19. Morales R, Rodriguez L, Singh A, Stratta E, Mendoza L, Valerio MA et al. National Survey of Medical Spanish Curriculum in U.S. Medical Schools. J Gen Intern Med (2015) 30(10):1434-1439. doi:10.1007/s11606-015-3309-3 
20. Mazor SS, Hampers LC, Chande VT, Krug SE.

Teaching Spanish to pediatric emergency physicians: effects of patient satisfaction. Arch Pediatr Adolesc Med (2002);156(7):693-695.

doi:10.1001/archpedi.156.7.693

21. Prince D, Nelson M. Teaching Spanish to emergency medicine residents. Acad Emerg Med (1995); 2(1):32-6. doi:10.1111/j.1553-

2712.1995.tb03076.x

22. Gonzalez SR, Gonzalez B, Moreira R, Ekdahl R, Vuk J. Needs assessment among students across colleges at UAMS for a Spanish interdisciplinary course: Directions for curriculum. AAMC Southern Group on Educational Affairs. Orlando, Florida. (2019).

23. Ríos J, Fernández Torres J, Rios TA, McGrawHill Education (Firm). Complete medical Spanish (2015). 